Pacific Journal of Mathematics

DIRECT SUMMANDS OF DIRECT PRODUCTS OF SLENDER 


\title{
DIRECT SUMMANDS OF DIRECT PRODUCTS OF SLENDER MODULES
}

\author{
JOHN D. O’NEILL
}

\begin{abstract}
Suppose $P=\prod_{I} G_{t}$ is a direct product of slender $R$-modules. If $|I|$ is non-measurable and $A$ is a direct summand of $P$, then $A \cong \prod_{J} A_{j}$ where each $A_{J}$ is isomorphic to a direct summand of a countable direct product of $G_{i}$ 's. If $R=Z$ and $P$ is a torsion-free reduced abelian group, then, if each $G_{\imath}$ has rank one, $A$ is a direct product of rank one groups.
\end{abstract}

1. Introduction. If $R$ is a ring and $M=\prod_{1}^{\infty} R_{n}$ with each $R_{n} \cong R$ as a $R$-module, then a $R$-module $N$ is slender if: for any homomorphism $f$ : $M \rightarrow N, f\left(R_{n}\right)=0$ for almost all $n$. In Theorem 3.7 we will show that, if a $R$-module $P$ equals $\Pi_{I} G_{i}$ with $|I|$ non-measurable and each $G_{i}$ slender, then any direct summand of $P$ is isomorphic to $\Pi_{j} A_{j}$ where each $A_{j}$ is isomorphic to a direct summand of a countable direct product of $G_{i}$ 's. This theorem in a way does for direct products what Kaplansky's theorem does for direct sums of modules (i.e., the theorem which states that projective modules are direct sums of countably generated modules [6]). In Theorem 4.3 we prove that if $V$ is a reduced vector group (a direct product of rank one torsion-free abelian groups) of non-measurable cardinality, then so is any direct summand of $V$. This answers Problem 74 in [4] for the non-measurable case.

2. Preliminaries. In this paper all groups are abelian, rings are associative with identity, modules are left unital, and homomorphisms are written on the left. Discussions of slender modules may be found in $[3,4$, 5, and 7]. For example, any torsion-free abelian group is a slender $Z$-module if it does not contain $Q, Z^{I}$ with $I$ infinite, or the $p$-adic integers for a prime $p$. This fact along with a good treatment of vector groups is contained in Chapter XIII of [4]. Unexplained terminology may be located in [4].

3. Slender modules. Throughout this section we shall consider the following situation. Let $R$ be a ring and let $R$-module $P$ equal $\Pi_{I} G_{i}=$ $A \oplus B$ where $|I|$ is non-measurable and each $G_{i}$ is slender. By $f_{i}, \alpha, \beta$ we shall mean the projections of $P$ to $G_{i}, A, B$ respectively and we let $\alpha_{\imath}=f_{i} \alpha$. 
Our first two lemmas are basic.

LEMMA 3.1.

(1) If $i$ is fixed, $\alpha_{t}\left(G_{j}\right)=0$ for almost all $j$ in $I$.

(2) If $i$ is fixed and $\alpha_{i}\left(G_{j}\right)=0$ for all $j$ in $J \subseteq I$, then $\alpha_{i}\left(\Pi_{J} G_{J}\right)=0$.

(3) If $\alpha_{t}\left(G_{J}\right)=0$ for all $j$ in $J$ and all $i$ in $I \backslash J$, then $\Pi_{J} G_{J}=\alpha\left(\Pi_{J} G_{J}\right)$ $\oplus \beta\left(\Pi_{J} G_{j}\right)$.

Proof. (1) follows from the definition of slender and (2) follows from Los' argument as given in the proof of Theorem 94.4 in [4] (see also the proof of Theorem 3 in [7] or Theorem 2.1 in [5]). The condition in (3) implies $\alpha\left(\Pi_{J} G_{j}\right)$ is contained in $\Pi_{J} G_{J}$ by (2). So $\beta\left(\Pi_{J} G_{j}\right) \subseteq \Pi_{J} G_{j}$ and (3) is true.

Lemma 3.2. (1) Let $D$ be any direct summand of $P$ and suppose $d_{j} \in D$ for each $j$ in a set $J$. If, for each fixed i in $I, f_{i}\left(d_{j}\right)=0$ for almost all $j$ in $J$, then the element $d=\Sigma_{I}\left(\sum_{J} f_{i}\left(d_{j}\right)\right)$ is in $D$. We define $d=\Sigma_{J} d_{J}$.

(2) Let $A_{j}$ be a submodule of $A$ for each $j$ in a set $J$ such that, for each $i \in I, f_{i}\left(A_{j}\right)=0$ for almost all $j$ in $J$. We define $\Sigma_{J} A_{J}=\left\{\Sigma_{J} a_{j} \mid a_{J} \in A_{j}\right\}$. Then $\sum_{J} A_{j}$ is in $A$ and $\sum_{J} A_{J} \cong \prod_{J} A_{j}$ if, whenever $\sum_{J} a_{J}=0$ with $a_{J} \in A_{J}$, then each $a_{j}=0$.

Proof. (1) We may suppose $D=B$ and just show $\alpha_{l}(d)=0$ for arbitrary $i$. By the previous lemma $\alpha_{i}\left(\Pi_{K} G_{k}\right)=0$ for some $K$ cofinite in $I$. For some subset $L$ cofinite in $J, \Sigma_{L} d_{J}$ is in $\prod_{K} G_{k}$. Since $d=\sum_{J \backslash L} d_{J}+$ $\Sigma_{L} d$, and the left sum is in $B, \alpha_{i}(d)=0$. (2) By (1) $\Sigma_{J} A_{J}$ is in $A$ and there is a natural isomorphism $\prod_{J} A_{j} \rightarrow \sum_{J} A_{J}$.

Note. The ideas in the first two lemmas will be used repeatedly without reference in the sequel.

Proposition 3.3. Suppose $J$ is a well-ordered set containing 1 and $A$ has submodules $A_{j}$ and $A^{J}$ for each $j$ in $J$ such that:

(1) $A=A^{1}$ and $A^{j}=A_{j} \oplus A^{J+1}$ (where $A^{J^{+1}}=0$ if $j$ is maximal in $J$ ),

(2) $A^{k}=\bigcap_{j<k} A^{j}$ if $k$ is a limit element in $J$,

(3) for each $i$ in $I f_{i}\left(A_{J}\right)=0$ for almost all $j$ in $J$,

(4) $\cap_{J} A^{j+1}=0$.

Then $A \cong \Pi_{J} A_{J}$.

Proof. By (3) $\sum_{J} A_{J}$ is a submodule of $A$. We need to show $A=\sum_{J} A_{j}$ and that, if $\sum_{J} a_{J}=0$ with $a_{j} \in A_{j}$, then each $a_{j}=0$. By our suppositions 
it will suffice to show:

$$
A=\left(\sum_{j \leq m} A_{j}\right) \oplus A^{m+1} \text { for each } m \text { in } J .
$$

Now $(*)$ is true for $m=1$ by (1) and we assume it is true for all $m<k$. If $k-1$ exists, $(*)$ is true for $k$ by (1). Suppose $k$ is a limit element in $J$. Let $a \in A$. By our assumption and (1) and (2) we may inductively choose $a, \in A_{j}$ for each $j<k$ so that $a-\left(a_{1}+\cdots+a_{j}\right) \in A^{j+1}$. Then $a-$ $\sum_{j<k} a_{j}$ is in $A^{i+1}$ for each $i<k$ and it is in $A^{k}$ by (2). By (1) then $a-\sum_{j \leq k} a_{j} \in A^{k+1}$ for some $a_{k} \in A_{k}$ and $A=\sum_{j \leq k} A_{j}+A^{k+1}$. Suppose $\sum_{j \leq k} a_{j}+x=0$ with $a_{j} \in A, x \in A^{k+1}$, and $a_{i} \neq 0$ for a minimal $i \leq k$. If $i=k, a_{i}=-x \in A^{i+1}$. If $i<k, a_{i} \in A^{i+1}$ by (*) for $m=i$. Either case implies $a_{t}=0$, a contradiction. So (*) is true for $m=k$ and by induction for all $m$.

Our next two lemmas deal with a particular ordering of $I$.

LEMMA 3.4. The set I can be ordered as an ordinal so that:

(1) for each $j$ in $I$, if $\alpha_{l}\left(G_{j}\right)=0$ for all $i<j$, then $\alpha_{i}\left(\Pi_{k \geq j} G_{k}\right)=0$ for all $i<j$,

(2) if $j$ is a limit ordinal in $I$, then $\alpha_{i}\left(\Pi_{k \geq j} G_{k}\right)=0$ for all $i<j$.

Proof. Let $1 \in \mathrm{I}$ be arbitrary. Suppose the ordinals $<m$ have been identified with $J$ a proper subset of $I$. Choose $m$ from $I \backslash J$ so that $\alpha_{k}\left(G_{m}\right) \neq 0$ for minimal $k$ in $J$ if possible; otherwise let $m$ from $I \backslash J$ be arbitrary. Continue in this manner until $I$ is totally ordered as an ordinal. This ordering implies (1) and we now show (2). Since $\alpha_{i}\left(G_{j}\right)=0$ for all $i<j$ if $j=1$, assume it is true for all non-successor ordinals $j$ less than limit ordinal $s$. Suppose $\alpha_{n}\left(G_{s}\right) \neq 0$ for some minimal $n<s$. Then $n-j$ is finite for $j=1$ or $j$ a limit ordinal $<s$. Let $K=\left\{i>n \mid \alpha_{k}\left(G_{i}\right) \neq 0\right.$ for some $k \leq n\}$. Since $\alpha_{k}\left(\Pi_{i \geq j} G_{i}\right)=0$ for all $k<j$ and since $\oplus_{j}^{n} G_{l}$ is slender, $K$ is finite. But $s$ is in $K$ and $s-n$ is finite by our ordering of $I$, a contradiction. Therefore (2) is true for $s$ and by induction for all limit ordinals.

Definition 3.5. Suppose $I$ is an ordinal and $J$ is a subset of $I$ containing 1. For each $j$ in $J$ let $j^{\prime}$ be the successor of $j$ in $J$ (if $j$ is maximal in $J$ let $j^{\prime}=I$ ). For each $j$ in $J$ set $I_{j}=\left\{i \in I \mid j \leq i<j^{\prime}\right\}$. Then $\left\{I_{j}\right\}$, $j \in J$, partitions $I$. Now let $P_{j}=\Pi_{I_{j}} G_{i}$ whence $P=\Pi_{J} P_{j}$. Also let $P^{j}=$ $\Pi_{l \geq j} G_{i}$. Then $P^{j}=P_{j} \oplus P^{j^{\prime}}$ (if $j$ is maximal in $J$ set $P^{J^{\prime}}=0$ ). 
LEMma 3.6. Suppose $I$ is an ordinal, $1 \in J \subseteq I$, and, for each $j \in J$, $\alpha_{i}\left(P^{j}\right)=0$ for all $i$ in $I$ less than $j$. Then $P_{j}=\alpha\left(P_{j}\right) \oplus \beta\left(P_{j}\right)$ for each $j$ and $A \cong \Pi_{J} \alpha\left(P_{j}\right)$.

Proof. Let $j \in J$ be arbitrary. By Lemma 3.1, $P^{j}=\alpha\left(P^{j}\right) \oplus \beta\left(P^{j}\right)$ and $P^{j^{\prime}}=\alpha\left(P^{j^{\prime}}\right) \oplus \beta\left(P^{j^{\prime}}\right)$. Therefore $P_{j}=\alpha\left(P_{j}\right) \oplus \beta\left(P_{j}\right)$. We now let $A_{j}=\alpha\left(P_{j}\right)$ and $A^{j}=\alpha\left(P^{j}\right)$ and apply Proposition 3.3 to show $A \cong \Pi_{J} A_{j}$. Since $1 \in J$ and $\alpha\left(P^{j}\right)=\alpha\left(P_{j}\right) \oplus \alpha\left(P^{j^{\prime}}\right),(1)$ is true. Suppose $k$ is in $J$ and a limit element therein. Then

$$
\begin{aligned}
A^{k} & =\alpha\left(P^{k}\right) \subseteq \bigcap_{j<k} \alpha\left(P^{j}\right)=\bigcap_{j<k} A^{j}=\alpha\left(\bigcap_{j<k} A^{j}\right) \\
& \subseteq \alpha\left(\bigcap_{j<k} P^{j}\right)=\alpha\left(P^{k}\right)=A^{k} .
\end{aligned}
$$

So (2) is true. Let $i \in I$ be fixed. From the map $\alpha_{i}: P=\Pi_{J} P_{J} \rightarrow G_{i}$ we see that $f_{i}\left(A_{j}\right)=\alpha_{i}\left(P_{j}\right)=0$ for almost all $j$. Hence (3) is true. Since $J$ is unbounded or $P^{j^{\prime}}=0$ for a maximal $j$ in $J, \cap A^{j^{\prime}} \subseteq \cap P^{j^{\prime}}=0$ and (4) is true. Therefore $A \cong \prod_{J} A_{j}$.

THEOREM 3.7. Suppose R-module $P$ equals $\Pi_{I} G_{i}=A \oplus B$ with $|I|$ non-measurable and each $G_{i}$ slender. Then $A \cong \Pi_{J} A_{j}$ where each $A_{j}$ is isomorphic to a direct summand of a countable direct product of $G_{i}$ 's.

Proof. Let $I$ be ordered as in 3.4 and let $J$ consist of 1 and all limit ordinals in $I$. For each $j \in J$ define $P^{j}$ and $P_{j}$ as in Definition 3.5 and set $A_{j}=\alpha\left(P_{j}\right)$. By 3.4, for each $j$ in $J, \alpha_{i}\left(P^{j}\right)=0$ for all $i$ in $I$ less than $j$. The theorem now follows from 3.6 and the fact that each $P_{J}$ is a countable product of $G_{i}$ 's.

4. Vector groups. A vector group is an abelian group of the form $V=\Pi_{I} R_{i}$ where each $R_{i}$ is torsion-free of rank one. Some twenty years ago (see [1]) it was shown that, if $V$ is reduced, $|I|$ is non-measurable, and $R_{i} \cong R_{j}$ or $\operatorname{Hom}\left(\operatorname{Hom}\left(R_{i}, R_{i}\right), R_{j}\right)=0$ for each $i$ and $j$, then any direct summand of $V$ is a vector group. We now remove the restrictions on the types of the $R_{i}$ 's. We thereby solve Problem 74 in [4] for the non-measurable case.

If $V$ above is reduced, it is a direct product of slender $Z$-modules; so the results in $\$ 3$ apply to it. Since $2^{\mu}$ is non-measurable for any non-measurable cardinal $\mu, V$ above has non-measurable cardinality if and only if $I$ has; so we equate these two properties henceforth. 
LEMMA 4.1. If $V=A \oplus B$ is a reduced vector group and $|V|$ is non-measurable, then there is a decomposition $V=\Pi_{I} R_{i}$ where each $R_{i}$ has rank one and type $t_{i}$ and, if $f_{i}, \alpha$ are the projections to $R_{i}, A$, respectively, and $\alpha_{i}=f_{i} \alpha$, then $\alpha_{i}\left(R_{j}\right)=0$ for each $i$ and $j$ unless $i=j$ or $t_{i}>t_{j}$.

Proof. Write $V=\Pi_{I} S_{i}$ with each $S_{i}$ of rank one and type $t_{i}$. Let $t$ be a type and set $V_{t}=\prod_{t_{t}=t} S_{i}$ and $V^{t}=\prod_{t_{t}>t} S_{i}$. By Lemma 96.1 in [4] $V^{t}$ and $V_{t} \oplus V^{t}$ are fully invariant subgroups of $V$. So $V^{t}=A^{t} \oplus B^{t}$ with $A^{t}$ in $A$ and $B^{t}$ in $B$. Also $V_{t} \oplus V^{t}=A_{t} \oplus B_{t} \oplus V^{t}$ with $A_{t}=A \cap\left(V_{t} \oplus B^{t}\right)$ and $B_{t}=B \cap\left(V_{t} \oplus A^{t}\right)$. If $\phi$ is the projection $\Pi_{I} S_{i} \rightarrow V_{t}$, then $V_{t}=\phi\left(A_{t}\right) \oplus$ $\phi\left(B_{t}\right)$ and each summand is a vector group by Theorem 1 in [1] (also exercise 10, p. 171, Vol. II of [4]). Thus, if $I_{t}=\left\{i \in I \mid t_{i}=t\right\}$, then $V_{t}$ has a decomposition $\prod_{I_{t}} R_{i}$, each $R_{i}$ of rank one, where, for each $i$ in $I_{t}$ and $x_{i}$ in $R_{i}$, there is a $y_{i}$ in $V^{t}$ such that $x_{i}=\left(x_{i}-y_{i}\right)+y_{i}$ with one term in $A$ and the other in $B$. Now, for some set $T$ of distinct types $t, V=\Pi_{T} V_{t}=$ $\Pi_{T}\left(\Pi_{I_{t}} R_{i}\right)=\Pi_{I} R_{i}$. By full invariance $\Pi_{t_{i}>t} R_{i}=V^{t}$ for each $t$. The conclusion of the lemma follows.

Our next lemma deals with a countable set of types.

LEMMA 4.2. Let $I$ be the natural numbers and let $T_{1}=\left\{t_{i}\right\}, i \in I$, be a set of types (not necessarily distinct). Let $I_{1}=\left\{i \in I \mid t_{i}\right.$ is maximal in $\left.T_{1}\right\}$. For each $n>1$ let $T_{n}=\left\{t_{i} \mid i \notin I_{1} \cup \cdots \cup I_{n-1}\right\}$ and $I_{n}=\left\{i \in I \mid t_{i}\right.$ is maximal in $\left.T_{n}\right\}$. Either (1) I contains an infinite chain $i_{1}<i_{2}<\cdots$ such that, for each $n, t_{i} \ngtr t_{i_{n}}$ whenever $i_{1} \leq i \leq i_{n}$ or (2) $I=\bigcup_{1}^{\infty} I_{n}$ and each $I_{n}$ is finite.

Proof. Suppose (2) is not true. Then, for some least $k, I_{n}$ is finite for $n<k$ and either $I_{k}$ is infinite or $T_{k}$ contains a chain of types not bounded above by an element in $T_{k}$. Let $i_{1}$ be an element in $I$ such that $i<i_{1}$ for all $i$ in $I_{n}, n<k$. Now $i_{1}$ satisfies the requirement in (1) and we assume $i_{1}, \ldots, i_{m}$ satisfy it. By our choice of $k$ and $i_{1}$ there is a $j>i_{m}$ such that $t_{i} \ngtr t_{j}$ whenever $i_{1} \leq i \leq i_{m}$. Let $i_{m+1}$ be the least such $j$. Then $t_{i} \ngtr t_{i_{m+1}}$ for $i_{1} \leq i \leq i_{m+1}$. Induction completes the proof.

THEOREM 4.3. If $V=A \oplus B$ is a reduced vector group and $|V|$ is non-measurable, then $A$ and $B$ are vector groups.

Proof. A proof for $A$ will suffice. Write $V=\prod_{I} R_{i}$ as in Lemma 4.1 and let $t_{i}, \alpha, \alpha_{i}$ be as defined there. Let $I$ be ordered as in 3.4 for $P=V$ and $G_{i}=R_{i}$. Thus for each $j$ in $I$, if $\alpha_{i}\left(R_{j}\right)=0$ for all $i<j$, then 
$\alpha_{i}\left(\Pi_{k \geq j} R_{k}\right)=0$ for all $i<j$ and, by the proof of Theorem 3.7, we may assume $I$ is the natural numbers. We now let $I_{n}$ and $T_{n}$ be as defined in 4.2 and treat the cases given there.

Case 1. There is an infinite sequence $i_{1}<i_{2}<\cdots$ in $I$ such that, for each $n, t_{i} \ngtr t_{i_{n}}$ for $i_{1} \leq i \leq i_{n}$. Since $\bigoplus_{i<i_{1}} R_{i}$ is slender, for some $m$, $\alpha_{i}\left(\Pi_{k \geq l_{m}} R_{k}\right)=0$ for all $i<i_{1}$. By our choice of $i_{n}$ 's and by 4.1 we must have, for each $n \geq m, \alpha_{i}\left(R_{i_{n}}\right)=0$ for all $i<i_{n}$. Therefore, from the way $I$ was ordered, for each $n \geq m, \alpha_{i}\left(\prod_{k \geq i_{n}} R_{k}\right)=0$ for all $i<i_{n}$. Let $J=$ $\left\{1, i_{m} i_{m+1}, \ldots\right\}$ and define $P^{j}$ and $P_{j}$ (with $G_{i}=R_{i}$ ) as in 3.5. By 3.6 then $A \cong \prod_{J} \alpha\left(P_{J}\right)$ and each $\alpha\left(P_{j}\right)$ is a direct summand of $P_{j}$. Since each $P_{j}$ is a finite rank vector group, so is each $\alpha\left(P_{J}\right)$. Therefore $V$ is a vector group.

Case 2. $I=\bigcup_{1}^{\infty} I_{n}$ and each $I_{n}$ is finite. We may assume $I$ is infinite. For each $n$ set $K_{n}=I_{1} \cup \cdots \cup I_{n}$ and let $V_{n}=\oplus_{K_{n}} R_{l}$ and $V^{n}=$ $\Pi_{I \backslash K_{n}} R_{i} . V_{n}$ is fully invariant in $V$ and equals $A_{n} \oplus B_{n}$ with $A_{n}$ in $A$ and $B_{n}$ in $B$. Also $A=A_{n} \oplus A^{n}$ where $A^{n}=A \cap\left(B_{n} \oplus V^{n}\right)$. We now find subgroups $C_{j}$ and $C^{j}$ in $A$ for $j \in J=(1,2, \ldots)$ such that:

(a) $A=C^{1}$ and $C^{j}=C_{j} \oplus C^{j+1}$,

(b) $C_{1} \oplus \cdots \oplus C_{j}=A_{m_{j}}$ for some $m_{j}$,

(c) $C^{J+2} \subseteq V^{j}$.

Let $C^{1}=A, C_{1}=A_{1}$, and $C^{2}=A^{1}$. The conditions are satisfied for $j=1$ by these $C$ 's and we assume they are satisfied for $j \leq k$ by the $C$ 's up to $C_{k}$ and $C^{k+1}$. Now $A=A_{m_{k}} \oplus C^{k+1}$ and $V=V_{k} \oplus V^{k}$. Since $A_{m_{k}}$ and $V_{k}$ are slender, from a consideration of projections: $V \rightarrow A \rightarrow A_{m_{k}}$ and $V \rightarrow A \rightarrow V_{k}$ we see that, for some large $n, \alpha\left(V^{n}\right)$ is in $C^{k+1}$ and $V^{k}$. For this $n$ then $A=A_{n} \oplus A^{n}$ where $A_{n} \supseteq A_{m_{k}}$ and

$$
A^{n}=A \cap\left(B_{n} \oplus V^{n}\right) \subseteq \alpha\left(V^{n}\right) \subseteq C^{k+1} \cap V^{k} .
$$

Let $C_{k+1}=A_{n} \cap C^{k+1}, C^{k+2}=A^{n}$, and $m_{k+1}=n$. Now $C^{k+1}=C_{k+1}$ $\oplus C^{k+2}, A_{m_{k}} \oplus C_{k+1}=A_{m_{k+1}}$, and $C^{k+2} \subseteq V^{k}$, as desired. Induction completes the sequences. Next we apply Proposition 3.3 to the subgroups $C_{\text {, and }} C^{J}$ with $j \in J$. Conditions (1) and (2) are clearly satisfied. Since $C_{j} \subseteq C^{J}$, (3) follows from (c) as does (4). So $A \cong \Pi_{J} C_{j}$. Since each $C_{j}$ is a finite rank vector group, $A$ is a vector group.

REMARK. This theorem cannot be improved. That is: a countably infinite direct product of rank two torsion-free groups can equal the direct sum of two indecomposable subgroups. An example of such a group can be constructed by modifying an infinite direct "sum" example of Corner (as found in [2] or Theorem 91.1 in [4]). This is explained more fully in [8]. 


\section{REFERENCES}

[1] S. Balcerzyk, A. Bialynicki-Birula and J. Los, On direct decompositions of complete direct sums of groups of rank 1, Bulletin de L'Academie Polonaise des Sciences, Vol. IX, No. 6, (1961), 451-454.

[2] A. L. S. Corner, A note on rank and direct decompositions of torsion-free abelian groups, II, Proc. Cambridge Philos. Soc., 66 (1969), 239-240.

[3] M. Dugas, and R. Gobel, Quotients of reflexive modules, Fundamenta Mathematicae, 114 (1981), 17-28.

[4] L. Fuchs, Infinite Abelian Groups, Vol. I (1970), Vol. II (1973), Academic Press, N. Y.

[5] M. Huber, On reflexive modules and abelian groups, J. Algebra, 82 (1983), 469-487.

[6] I. Kaplansky, Projective modules, Ann. Math., 68 (1958), 372-377.

[7] E. Lady, Slender rings and modules, Pacific J. Math., 49 (1973), 397-406.

[8] J. D. O’Neill, On summands of Direct Products of Abelian Groups, Comment. Math. Univ. Carolinae, 24.3 (1983), 407-413.

Received April 14, 1983 and in revised form September 13, 1983.

UNIVERSITY OF DETROIT

DETROIT, MI 48221 



\section{PACIFIC JOURNAL OF MATHEMATICS EDITORS}

DONALD BABBITT (Managing Editor)
University of California
Los Angeles, CA 90024
CHARLES R. DEPrIMA
California Institute of Technology
Pasadena, CA 91125
R. FINN
Stanford University
Stanford, CA 94305

\author{
HERMANN FLASCHKa \\ University of Arizona \\ Tucson, AZ 85721 \\ RAMESH A. GANGOLLI \\ University of Washington \\ Seattle, WA 98195 \\ ROBION KIRBY \\ University of California \\ Berkeley, CA 94720 \\ C. C. Moore \\ University of California \\ Berkeley, CA 94720
}

Hugo Rossi

University of Utah

Salt Lake City, UT 84112

H. SAMELSON
Stanford University
Stanford, CA 94305

HAROLD STARK

University of California, San Diego

La Jolla, CA 92093

\section{ASSOCIATE EDITORS}
R. ARens
E. F. BECKENBACH (1906-1982)
B. H. NeUmanN
F. WOLF
K. YOSHIDA

\begin{tabular}{ll}
\multicolumn{2}{c}{ SUPPORTING INSTITUTIONS } \\
UNIVERSITY OF ARIZONA & UNIVERSITY OF OREGON \\
UNIVERSITY OF BRITISH COLUMBIA & UNIVERSITY OF SOUTHERN CALIFORNIA \\
CALIFORNIA INSTITUTE OF TECHNOLOGY & STANFORD UNIVERSITY \\
UNIVERSITY OF CALIFORNIA & UNIVERSITY OF HAWAII \\
MONTANA STATE UNIVERSITY & UNIVERSITY OF TOKYO \\
UNIVERSITY OF NEVADA, RENO & UNIVERSITY OF UTAH \\
NEW MEXICO STATE UNIVERSITY & WASHINGTON STATE UNIVERSITY \\
OREGON STATE UNIVERSITY & UNIVERSITY OF WASHINGTON
\end{tabular}

The Supporting Institutions listed above contribute to the cost of publication of this Journal, but they are not owners or publishers and have no responsibility for its content or policies.

\footnotetext{
Mathematical papers intended for publication in the Pacıfic Journal of Mathematıcs should be in typed form or offset-reproduced (not dittoed), double spaced with large margins. Please do not use built up fractions in the text of the manuscript. However, you may use them in the displayed equations. Underline Greek letters in red, German in green, and script in blue. The first paragraph must be capable of being used separately as a synopsis of the entire paper. In particular it should contain no bibliographic references. Please propose a heading for the odd numbered pages of less than 35 characters. Manuscripts, in triplicate, may be sent to any one of the editors. Please classify according to the scheme of Math. Rev ews, Index to Vol. 39. Supply name and address of author to whom proofs should be sent. All other communications should be addressed to the managing editor, or Elaine Barth, University of California, Los Angeles, California 90024.

There are page-charges associated with articles appearing in the Pacific Journal of Mathematics. These charges are expected to be paid by the author's University, Government Agency or Company. If the author or authors do not have access to such Institutional support these charges are waived. Single authors will receive 50 free reprints; joint authors will receive a total of 100 free reprints. Additional copies may be obtained at cost in multiples of 50 .
}

The Pacific Journal of Mathematics is issued monthly as of January 1966. Regular subscription rate: $\$ 190.00$ a year (5 Vols., 10 issues). Special rate: $\$ 66.00$ a year to individual members of supporting institutions.

Subscriptions, orders for numbers issued in the last three calendar years, and changes of address should be sent to Pacific Journal of Mathematics, P.O. Box 969, Carmel Valley, CA 93924, U.S.A. Old back numbers obtainable from Kraus Periodicals Co., Route 100, Millwood, NY 10546.

The Pacific Journal of Mathematics at P.O. Box 969, Carmel Valley, CA 93924 (ISSN 0030-8730) publishes 5 volumes per year. Application to mail at Second-class postage rates is pending at Carmel Valley, California, and additional mailing offices. Postmaster: Send address changes to Pacific Journal of Mathematics, P.O. Box 969, Carmel Valley, CA 93924.

PUBLISHED BY PACIFIC JOURNAL OF MATHEMATICS, A NON-PROFIT CORPORATION Copyright $\odot 1985$ by Pacific Journal of Mathematics 


\section{Pacific Journal of Mathematics}

\section{Vol. 117, No. 2 \\ February, 1985}

Robert Walter Bagley, Ta-Sun Wu and J. S. Yang, On a class of topological groups more general than SIN groups ............... 209

Bruce Alan Barnes, Algebraic elements of a Banach algebra modulo an ideal ...............................................219

Howard D. Fegan and Peter Gilkey, Invariants of the heat equation .......233

Erica Flapan, Necessary and sufficient conditions for certain homology

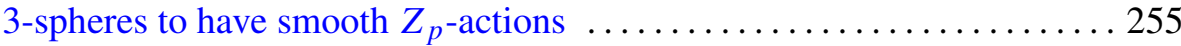

Philip R. Heath, Product formulae for Nielsen numbers of fibre maps . . . . 267

Derbiau Frank Hsu and A. Donald Keedwell, Generalized complete mappings, neofields, sequenceable groups and block designs. II ...... 291

Taqdir Husain, Orthogonal primitive idempotents and Banach algebras

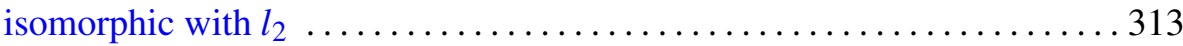

Gary M. Lieberman, Regularized distance and its applications . . . . . . . 329

William W. Menasco, Determining incompressibility of surfaces in alternating knot and link complements ............................ 353

Benjamin Muckenhoupt, Weighted reverse weak type inequalities for the Hardy-Littlewood maximal function $\ldots \ldots \ldots \ldots \ldots \ldots \ldots \ldots \ldots \ldots \ldots \ldots$

John Dacey O'Neill, Direct summands of direct products of slender

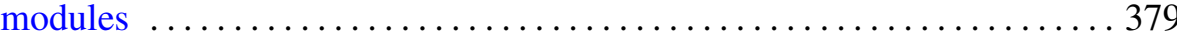

Andreas Rapp, Elimination of Malitz quantifiers in stable theories 387

Francisco José Ruiz, A unified approach to Carleson measures and $A_{p}$ weights

Hanamatagouda Pandappa Sankappanavar, Heyting algebras with dual

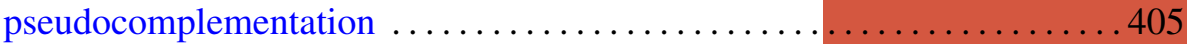

Annette Sinclair, Strong Carleman and strong uniform approximation . . . . 417

Masaaki Suzuki, The generalized Schwarz lemma for the Bergman metric

Brian Thorpe and Ludwig Tomm, Universal approximation by regular

weighted means 